\title{
Quantification of Genotoxic Impurity 4-Chloro-1-Hydroxy Butane Sulfonic Acid Sodium Salt by LCMS/MS in Sumatriptan Succinate
}

\author{
Narayana MBV ${ }^{1 *}$, Chandrasekhar $\mathrm{KB}^{2}$ and $\mathrm{Rao}^{\mathrm{B}} \mathrm{M}^{3}$
}

${ }^{1}$ Analytical Research, Matrix Laboratories limited, Hyderabad, India

${ }^{2}$ Department of Chemistry, Jawaharlal Nehru Technological University of College of Engineering, Anantapur 515002, India

${ }^{3}$ Analytical Research, Johnson and Johnson Ltd., Mumbai-400080, India

\begin{abstract}
The objective of present research work is to develop a suitable LCMS/MS method for the quantitative determination of genotoxic impurity 4-Chloro-1-Hydroxy Butane Sulfonic Acid Sodium Salt at ppm level present in Sumariptan dug substance. The LCMS/MS method was developed on Zorbax SB-C8 column using the mobile phase consists a mixture of $0.05 \%(\mathrm{v} / \mathrm{v})$ Formic acid in water and Acetonirile using a isocratic composition of 90:10 $(\mathrm{v} / \mathrm{v})$ at a flow rate of $0.8 \mathrm{~mL} / \mathrm{min}$. lon source is electospray ionization $(\mathrm{ESI})$, source temperature is $325^{\circ} \mathrm{C}$, gas flow is $8 \mathrm{~L} / \mathrm{min}$, Nebuliser pressure is $40 \mathrm{psi}$, capillary voltage is $4000 \mathrm{~V}$. Under these conditions impurity was quantified by selecting most stable MRM pair (187/81). The limit of detection and the limit of quantitation for the impurity were established. Validation of the developed LCMS/MS method was carried out as per ICH requirements and the data shows that the proposed method is specific, linear, accurate, precise and robust. This method has been tested in a number of Sumatriptan samples and used successfully for quantification of the impurity at ppm level. The developed LCMS/MS method was found to be suitable to quantify the genotoxic impurity 4-Chloro-1-Hydroxy Butane Sulfonic Acid Sodium Salt at ppm level present in Sumatriptan Succinate.
\end{abstract}

Keywords: Genotoxic impurity; Liquid Chromatography Mass Spectrometry (LCMS); 4-Chloro-1-Hydroxy Butane sulfonic acid sodium salt; ppm (parts per million); Sumatriptan; Threshold of Toxicological Concern (TTC)

\section{Introduction}

4-Chloro-1-Hydroxy Butane Sulfonic Acid Sodium Salt is an impurity during the synthesis of Sumatriptan succinate. The impurity is found to be genotoxic because it contains two functional groups primary alkyl halide [1] and Sulfonate which causes for the genotoxicity [2-5]. Genotoxic substances are chemicals that harm an organism by damaging its genetic material (DNA). Specifically, there is evidence that genotoxic substances may bind directly to DNA and may also act indirectly by affecting enzymes involved in DNA replication. There are three primary effects that genotoxins can have on organisms by affecting their genetic information. Genotoxins can be carcinogens, or cancer-causing agents, mutagens, or mutation-causing agents, or teratogens, birth defect-causing agents [6]. The toxicological assessment of these genotoxic impurities and the determination of acceptable limits for such impurities in active substances is a difficult issue and not addressed in sufficient detail in the existing International Conference on Harmonization (ICH) Q3X guidelines [7]. The presence of trace level of the impurity in drug substance or drug product is of genotoxicity concern and has been closely scrutinized by regulatory agencies and pharmaceutical industries [8]. The 'threshold of toxicological concern' (TTC) of $1.5 \mu \mathrm{g} /$ person/day (exposure of genotoxic impurity in drugs that will be tested or dosed for longer than 12 months) has been suggested by the European Medicines Agency's (EMEA) "Guideline on the limits of genotoxic impurities" [9-12] and the Pharmaceutical Research and Manufacturers of America's (PhRMA) white paper [13]. Based on the TTC, the concentration limits of genotoxic impurity in drug substances or drug products can then be derived based on the maximum daily dose: concentration limit (ppm) $=[1.5 \mu \mathrm{g} /$ day $)] /[$ dose $(\mathrm{g} /$ day $)]$. For a drug dosed at $1 \mathrm{~g}$ per day, for example, $1.5 \mathrm{ppm}$ would be the limit of a specific genotoxic impurity which would also be the 'target analyte level' (TAL) from an analytical perspective [9-12]. Given such a low ppm concentration limit, besides the control challenges in process chemistry, developing sensitive and robust methodology for their detection poses a tremendous analytical challenge for the pharmaceutical industry $[14,15]$. Therefore potential genotoxins must be minimized during the synthesis the compounds and where there is difficulty achieving this, the method of manufacture should preferably be changed [1]. As 4-Chloro-1-Hydroxy Butane Sulfonic Acid Sodium Salt is a genotoxic compound, the regulators may require the toxin levels to be controlled to $2 \mathrm{ppm}$ in the drug substance. Quantification at such very low level can be possible only by using LCMS/MS and also there is no method for the quantification of this impurity hence a high sensitive LCMS/MS method developed for the quantification of this genotoxic impurity 4-Chloro-1-Hydroxy Butane Sulfonic Acid Sodium Salt.

\section{Experimental}

\section{Chemicals and reagents}

Samples of Sumatriptan Succinate and 4-Chloro-1-Hydroxy Butane Sulfonic Acid Sodium Salt (Figure 1) were received from Bulk Actives, Unit-II of Aurabindo Laboratories, Hyderabad, India. HPLC grade Acetonitrile was purchased from J T Baker, Mumbai, India. Formic acid was purchased from Sigma Aldrich, Mumbai, India. High pure water was prepared by using Millipore Milli Q plus purification system (Millipore, USA).

*Corresponding author: Narayana MBV, Analytical Research, Matrix Laboratories limited, Hyderabad 500 072, India, E-mail: balajimachireddy@gmail.com

Received November 27, 2012; Accepted December 26, 2012; Published December 31, 2012

Citation: Narayana MBV, Chandrasekhar KB, Rao BM (2012) Quantification of Genotoxic Impurity 4-Chloro-1-Hydroxy Butane Sulfonic Acid Sodium Salt by LCMS/ MS in Sumatriptan Succinate. J Bioanal Biomed 4: 104-107. doi:10.4172/1948593X.1000072

Copyright: ( 2012 Narayana MBV, et al. This is an open-access article distributed under the terms of the Creative Commons Attribution License, which permits unrestricted use, distribution, and reproduction in any medium, provided the original author and source are credited. 
<smiles>CNS(=O)(=O)Cc1ccc2[nH]cc(CCN(C)C)c2c1</smiles><smiles>O=C(O)CCC(=O)O</smiles>

Succinate: 1-[3-(2-dimethylaminoethyl)-1H-indol-5-yl]-N-methyl-methanesulfonamide.<smiles>N#[V]C(O)CCCCl</smiles>

Impurity: 4-Chloro-1-Hydroxy Butane Sulfonic Acid Sodium Salt

Figure 1: Structures of Samaritan Succinate and its genotoxic impurity.

\section{Equipment}

The LCMS method development and validation were done using Agilent 1200 series HPLC system Connected with Agilent mass spectrometer LCMS/MS-QqQ system (Agilent technologies, Germany) equipped with Electro spray ionization probe. The data were collected using Agilent mass hunter work station software.

\section{LCMS chromatographic conditions}

The LC chromatographic separations were achieved on Zorbax SC-C8 column $150 \mathrm{~mm}$ length $\times 4.6 \mathrm{~mm}$ ID with $5 \mu \mathrm{m}$ particle size using the isocratic mobile phase of mixture of $0.05 \%(\mathrm{v} / \mathrm{v})$ Formic acid in water and acetonitrile using a isocratic composition of 90:10 $(\mathrm{v} / \mathrm{v})$ at a flow rate of $0.8 \mathrm{~mL} / \mathrm{min}$. Mass spectrometer was operated in electospray ionization (ESI) negative ion mode with a capillary voltage of $4000 \mathrm{~V}$. The fragmentor was set at $70 \mathrm{~V}$, the drying gas flow was 8 $\mathrm{L} / \mathrm{min}$ with a temperature of $325^{\circ} \mathrm{C}$ and nebuliser pressure was 40 psi. Under these conditions impurity was quantified by selecting high sensitive stable Multi reaction monitoring (MRM) ion pair $187 \rightarrow 81$. The test concentration was about $100 \mathrm{mgmL}^{-1}$ and the injection volume was $20 \mu \mathrm{L} .0 .1 \%$ formic acid in water was used as diluent during the standard and test samples preparations.

\section{Preparation of impurity standard and test sample Solution}

The stock solution of impurity standard prepared at approximately $1 \mathrm{mgmL}^{-1}$ in pure diluent. For linearity, the stock solution impurity was diluted using diluent to give standards at $0.5,0.7,1.0,1.2,1.5 \mathrm{ppm}$ with respect to test concentration. The testing API samples were typically prepared at approximately $100 \mathrm{mg} / \mathrm{mL}$ in diluent and sonicated about 10 minutes and filtered through $0.45 \mu$ poly tetrafluoroethylene (PTFE) filter.

\section{Results and Discussion}

\section{Optimization of chromatographic conditions}

The main target of LC-MS/MS method was to quantify the 4-Chloro-1-Hydroxy Butane Sulfonic Acid Sodium Salt in the Sumatriptan succinate drug substance. As volatile buffers required for analysis in LCMS the mobile phase was restricted to volatile buffers like Formic acid, trifluoro acetic acid up to $0.05 \%$ level, ammonium acetate up to $5 \mathrm{mM}$ concentration. As formic acid is the most suitable buffer to get more sensitivity, by using a mixture of $0.05 \%$ formic acid in water and acetonitrile in the ratio of $80: 20(\mathrm{v} / \mathrm{v})$ at a flow rate of $1.0 \mathrm{~mL} / \mathrm{min}$ as mobile phase and SB-C18 column, impurity spiked sample injected, impurity was eluting with less retention and resolution between drug and impurity is very less. Then with same mobile composition SB-C8 column was tried and the impurity slightly separated from the drug substance. To get further separation the mobile phase composition changed to $90: 10(\mathrm{v} / \mathrm{v})$ at a flow rate of $0.8 \mathrm{~mL} / \mathrm{min}$ with same column SB-C8 in this condition the impurity is well separated with good peak shape from the drug substance. As the MRM in LCMS will give more sensitive quantization, hence to quantify by using this mode the molecule should have intense fragmented ion. The present impurity has given three fragments out of which the most intense fragment ion was 81 used for quantification. By selecting this MRM pair $18 \rightarrow 781$ and the above chromatographic conditions the optimized mass parameters are fragmentor voltage $70 \mathrm{~V}$, the drying gas flow was $8 \mathrm{~L} / \mathrm{min}$ with a drying temperature of $325^{\circ} \mathrm{C}$ and nebulizer pressure was $40 \mathrm{psi}$.

\section{Method Validation}

\section{Linearity}

The linearity of 4-Chloro-1-Hydroxy Butane Sulfonic Acid Sodium Salt was satisfactorily done. A series of solutions were prepared using 4-Chloro-1-Hydroxy Butane Sulfonic Acid Sodium Salt at concentration levels from around detection level to $150 \%$ and the concentration levels are $0.5,0.7,1.0,1.2,1.5 \mathrm{ppm}$ respectively. The peak area versus concentration data was done by linearity plot slope, intercept, and residual sum of squares analysis. The calibration curve was given based on response over the concentration range for 4-Chloro-1Hydroxy Butane Sulfonic Acid Sodium Salt. The correlation coefficient 4-Chloro-1-Hydroxy Butane Sulfonic Acid Sodium Salt was 0.996 and the Linearity results are tabulated in table 1.

\section{Limit of Detection (LOD) and Limit of Quantitation (LOQ)}

The LOD and LOQ values of 4-Chloro-1-Hydroxy Butane Sulfonic Acid Sodium Salt were predicted from the linearity data. Each predicted concentration was verified for precision by preparing the solutions at about predicted concentration and injecting each solution six times for

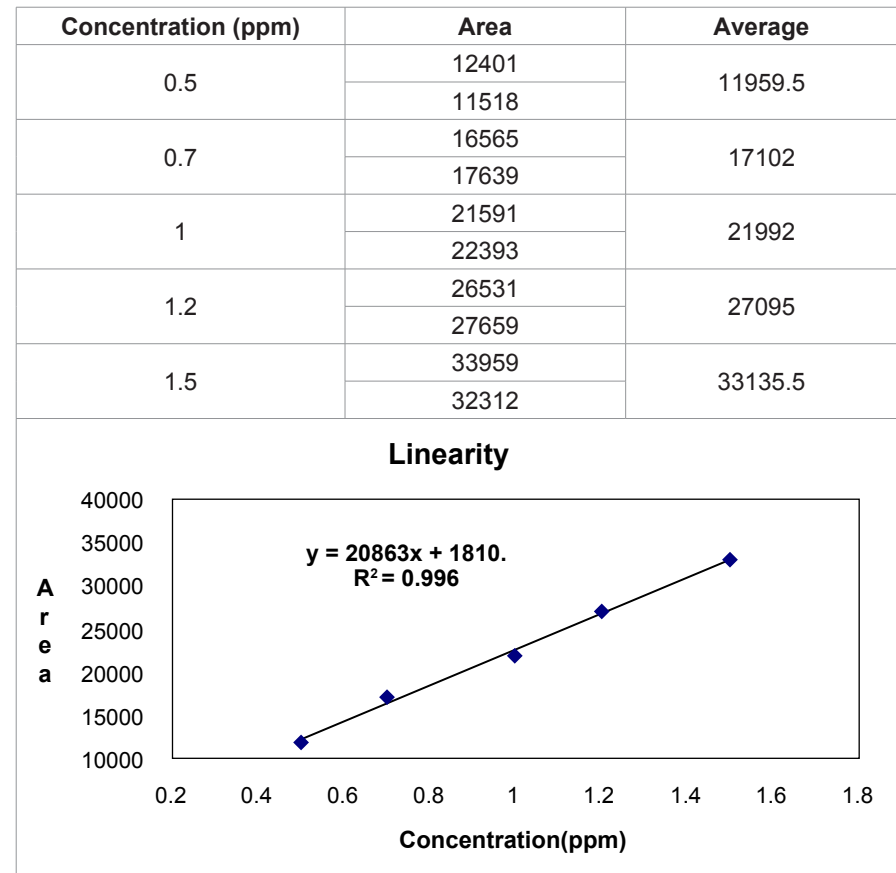

Table 1: Results of Linearity. 
LC-MS/MS study and the predicted concentration for LOQ was 0.5 ppm and LOD was $0.17 \mathrm{ppm}$ (Figure 2) and the results are tabulated in table 2.

\section{Precision}

The precision of the developed method was checked by preparing solutions by spiking the impurity at LOQ, $100 \%$ and $150 \%$ level with the drug substance for six times and injected each once also injected $100 \%$ spiked solution for 6 times to show the system precision. The $\%$ relative standard deviation (RSD) of the areas at each level 5.7\%, 2.4\% and $2.7 \%$ confirming the good precision of the developed method.

\section{Accuracy}

The accuracy of the method was evaluated in sample solutions
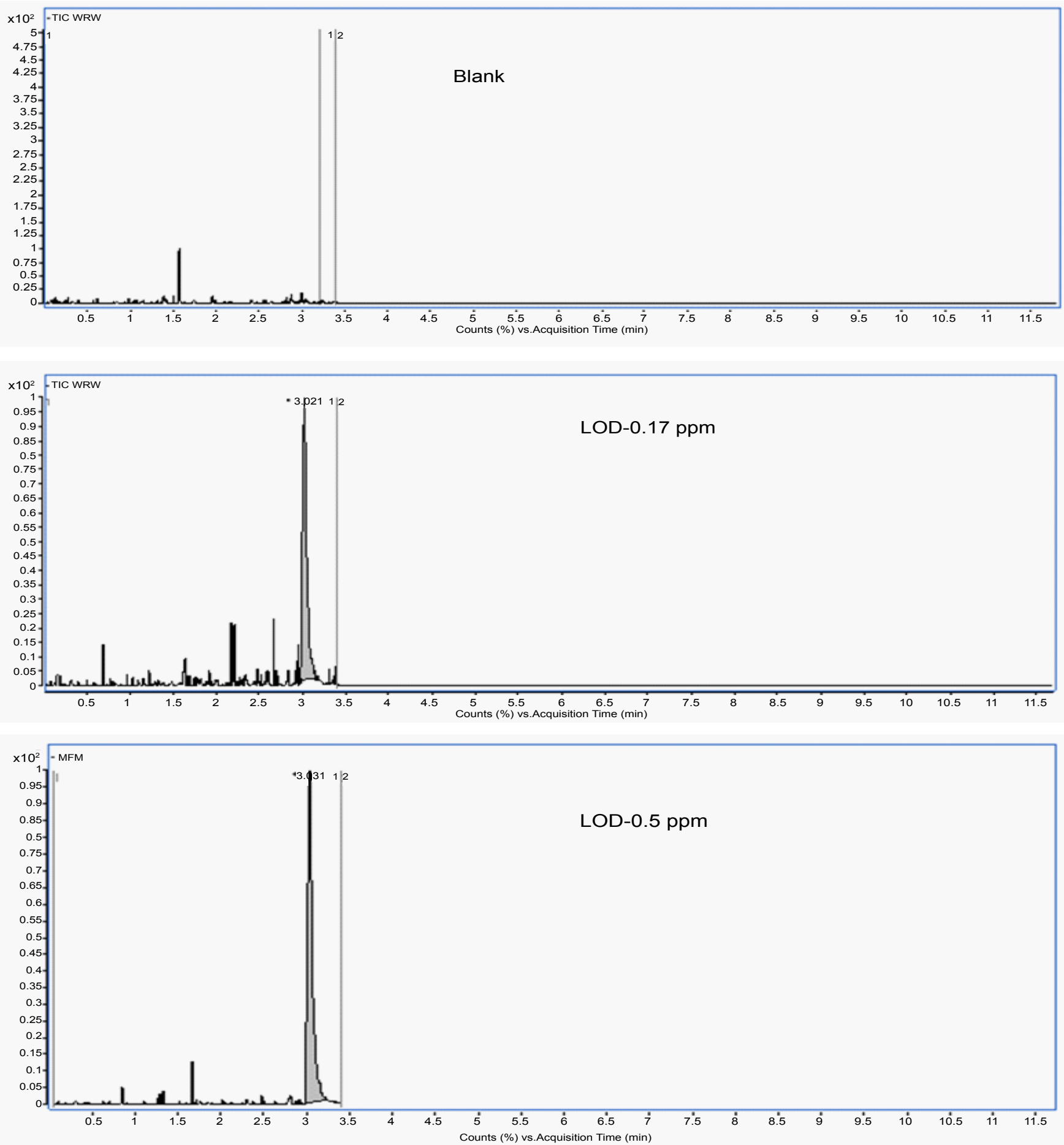

Figure 2: Typical mass spectrograms of blank, LOD, LOQ. 
Citation: Narayana MBV, Chandrasekhar KB, Rao BM (2012) Quantification of Genotoxic Impurity 4-Chloro-1-Hydroxy Butane Sulfonic Acid Sodium Salt by LCMS/MS in Sumatriptan Succinate. J Bioanal Biomed 4: 104-107. doi:10.4172/1948-593X.1000072

\begin{tabular}{|c|c|c|}
\hline \multirow{2}{*}{ Injection } & \multicolumn{2}{|c|}{ Area } \\
\cline { 2 - 3 } & LOD (0.7 $\mathbf{~ p m )}$ & LOQ $\mathbf{( 0 . 5} \mathbf{~ p m )}$ \\
\hline 1 & 5500 & 18850 \\
\hline 2 & 4810 & 16931 \\
\hline 3 & 5391 & 17156 \\
\hline 4 & 5269 & 17529 \\
\hline 5 & 5455 & 17819 \\
\hline 6 & 4710 & 18399 \\
\hline Average & 5189 & 17781 \\
\hline SD & 343 & 735 \\
\hline \%RSD & 6.6 & 4.13 \\
\hline
\end{tabular}

SD: Standard Deviation, RSD: Relative Standard Deviation

Table 2: Results of LOD and LOQ Precision.

\begin{tabular}{|c|c|c|c|c|c|c|}
\hline Level & $\begin{array}{c}\text { Amount } \\
\text { Added }(\mu \mathrm{g})\end{array}$ & $\begin{array}{l}\text { Amount } \\
\text { found }(\mu \mathrm{g})\end{array}$ & $\%$ Recovery & Mean & SD & $\%$ RSD \\
\hline LOQ Sample-1 & \multirow{3}{*}{0.512} & 0.479 & 93.6 & \multirow{3}{*}{97.3} & \multirow{3}{*}{4.7} & \multirow{3}{*}{4.9} \\
\hline LOQ Sample-2 & & 0.525 & 102.6 & & & \\
\hline LOQ Sample-3 & & 0.489 & 95.6 & & & \\
\hline 75\% Sample-1 & \multirow{3}{*}{0.767} & 0.729 & 95.0 & \multirow{3}{*}{94.2} & \multirow{3}{*}{1.0} & \multirow{3}{*}{1.1} \\
\hline 75\% Sample-2 & & 0.726 & 94.6 & & & \\
\hline 75\% Sample-3 & & 0.714 & 93.1 & & & \\
\hline $100 \%$ Sample-1 & \multirow{3}{*}{1.023} & 1.015 & 99.2 & \multirow{3}{*}{97.2} & \multirow{3}{*}{2.4} & \multirow{3}{*}{2.5} \\
\hline $100 \%$ Sample-2 & & 1.001 & 97.8 & & & \\
\hline $100 \%$ Sample-3 & & 0.967 & 94.5 & & & \\
\hline $125 \%$ Sample-1 & \multirow{3}{*}{1.279} & 1.237 & 96.7 & \multirow{3}{*}{98.4} & \multirow{3}{*}{4.1} & \multirow{3}{*}{4.2} \\
\hline $125 \%$ Sample-2 & & 1.219 & 95.3 & & & \\
\hline $125 \%$ Sample-3 & & 1.318 & 103.1 & & & \\
\hline $150 \%$ Sample-1 & \multirow{3}{*}{1.535} & 1.401 & 91.3 & \multirow{3}{*}{95.5} & \multirow{3}{*}{3.7} & \multirow{3}{*}{3.9} \\
\hline $150 \%$ Sample-2 & & 1.511 & 98.5 & & & \\
\hline $150 \%$ Sample-3 & & 1.485 & 96.8 & & & \\
\hline
\end{tabular}

SD: Standard Deviation, RSD: Relative Standard Deviation

Table 3: Results of Accuracy study.

were prepared in triplicate by spiking 4-Chloro-1-Hydroxy Butane Sulfonic Acid Sodium Salt at LOQ level, 75\%, 100\%, 125\% and 150\% with Sumatriptan succinate and injected each solution in to LCMS as per methodology. The percentage of recovery for the impurity was calculated and the values are $97.3 \%, 94.2 \%, 97.2,98.4$, and $95.5 \%$. At such low levels these recoveries and \% relative standard deviation (RSD) were satisfactory and the results are tabulated in table 3.

\section{Conclusion}

In this paper a sensitive specific, accurate, validated and welldefined LCMS/MS method for the Quantification of genotoxic impurity 4-Chloro-1-Hydroxy Butane Sulfonic Acid Sodium Salt at ppm level in Sumatriptan succinate was described. The limit of detection and limit of quantification found to be $0.17 \mathrm{ppm}$ and $0.5 \mathrm{ppm}$ respectively. The described method is highly reliable technique for the quantification of the gentoxic impurity present in the sumatriptan succinate during quality control testing.

\section{Acknowledgments}

The authors wish to thank the professors and research Scholars for supporting this work. We would also like to thank colleagues in separation science division of Analytical Research Matrix Laboratories Limited for their cooperation in carrying out this work.

\section{References}

1. Elder DP, Lipczynski AM, Teasdale A (2008) Control and analysis of alkyl and benzyl halides and other related reactive organohalides as potential genotoxic impurities in active pharmaceutical ingredients (APIs). J Pharm Biomed Anal 48: 497-507.

2. Genotoxic and Carcinogenic Impurities in Drug Substances and Products: Recommended Approaches (2008) US Department of Health and Human Services, Food and Drug Administration, Center for Drug Evaluation and Research CDER USA.

3. Muller L, Gocke E (2009) Considerations regarding a permitted daily exposure calculation for ethyl methanesulfonate. Toxicol Lett 190: 330-332.

4. Liu DQ, Sun M, Kord AS (2010) Recent advances in trace analysis of pharmaceutical genotoxic impurities. J Pharm Biomed Anal 51: 999-1014.

5. Dobo KL, Greene N, Cyr MO, Caron S, Ku WW (2006) The application of structure-based assessment to support safety and chemistry diligence to manage genotoxic impurities in active pharmaceutical ingredients during drug development. Regul Toxicol Pharmacol 44: 282-293.

6. Yuabova, Holschlag ZY, Rodriguez DR, Qin SA, Papov C, et al. (2008) Genotoxic Impurities: A Quantitative Approach. J Liq Chromatogr Relat Technol 31: 2318-2330.

7. Guideline on Impurities in New Drug Substances, Q3A (R2), ICH.

8. Muller L, Mauthe RJ, Riley CM Andino MM, Antonis DD et al (2006) A rationale for determining, testing, and controlling specific impurities in pharmaceuticals that possess potential for genotoxicity. Regul Toxicol Pharmacol 44: 198-211.

9. http://www.ema.europa.eu/docs/en_GB/document_library/Scientific guideline/2009/09/WC500002903.pdf

10. Kroes R, Renwick AG, Cheeseman M, Kleiner J, Mangelsdorf I, et al. (2004) Structure-based thresholds of toxicological concern (TTC): guidance for application to substances present at low levels in the diet. Food Chem Toxicol 42: 65-83.

11. McGovern T, Jacobson KD (2006) Regulation of genotoxic and carcinogenic impurities in drug substances and drug products. Trends Analyt Chem 25:790795.

12. Bass R, Purves J, Amati MP (2000) Safety of biotechnological products Pharmacol Toxicol 86: 27-29.

13. Delaney EJ (2007) An impact analysis of the application of the threshold of toxicological concern concept to pharmaceuticals. Regul Toxicol Pharmacol 49: 107-124.

14. Colon I, Richoll SM (2005) Determination of methyl and ethyl esters of methanesulfonic, benzenesulfonic and p-toluenesulfonic acids in active pharmaceutical ingredients by solid-phase microextraction (SPME) coupled to GC/SIM-MS. J Pharm Biomed Anal 39: 477-485.

15. Li H, Sluggett GW (2005) Development and validation of a sensitive GC-MS method for the determination of trace levels of an alkylating reagent in a betalactam active pharmaceutical ingredient. J Pharm Biomed Anal 39: 486-494. 